\title{
On the morphology of ammonium nitrate (III): theory and observation
}

\author{
L.J.P. Vogels, P. Bennema, M.H.J. Hottenhuis * and M.C. Elwenspoek ** \\ RIM Laboratory of Solid State Chemistry, University of Nijmegen, Toernooiveld, 6525 ED Nijmegen, Netherlands
}

Received 25 February 1990; manuscript received in final form 24 August 1990

The aim of this paper is to derive on a theoretical basis the morphology of crystals of ammonium nitrate, phase III. and to compare the results with experimental growth forms. The theory used is based on the concepts of periodic bond chain (PBC), F face and connected net, developed by Hartman and Perdok. Further an Ising model is used to determine roughening temperatures. Based on different criteria theoretical growth forms are predicted and compared with experiments.

\section{Introduction}

Ammonium nitrate is an important fertilizer and explosive of which vast amounts are produced yearly. Since the last century it has been object for many investigations [1-3]. It is a polymorphic salt with five different structures under atmospheric conditions, of which the crystal structure of phase I, II and IV are similar to each other, while phase III differs [4]:

$$
\begin{aligned}
& \mathrm{I}-(T=498.2 \mathrm{~K})-\mathrm{II}-(T=457.2 \mathrm{~K})- \\
& \text { III- }(T=305.2 \mathrm{~K})-\mathrm{IV}-(T=355.5 \mathrm{~K})-\mathrm{V} .
\end{aligned}
$$

At room temperature phase IV is stable, while above $305.2 \mathrm{~K}$ phase III becomes stable. Stress and crystallographic properties make the phase transition between these two phases very difficult. Therefore the phase transition IV $\rightarrow$ III $\rightarrow$ II only occurs in moist samples [5]. In "dry" samples phase IV becomes metastable and transforms at about $328 \mathrm{~K}$ into a metastable phase II [5-7]. During the phase transition IV $\rightarrow$ III the crystal recrystallizes partly by dissolving and partly by

\footnotetext{
* Present address: AKZO Research Laboratories, P.O. Box 9300,6800 SB Arnhem, The Netherlands.

** Present address: Faculty of Electrotechnics, Technical University Twente, P.O. Box 217, 7500 AE Enschede, The Netherlands.
}

forming new domains. The volume of the unit cell increases with $4 \%$, causing serious density problems, thus a fertilizer becomes an explosive. Also severe straining and caking was found [6]. The rate of transformation may be described by assuming that the growth of nuclei is rate determining, but there is a serious disagreement in rate velocities reported [8-13]. It is believed that the morphology of crystals of phases III and IV determines to a high extent the properties of this chemico-physical transformation [5-7]. This paper reports on a study of the morphology of ammonium nitrate phase III. In order to check the predictions of the theory, the morphology of $\mathrm{NH}_{4} \mathrm{NO}_{3}$ is determined for the first time from goniometric investigations of about ten crystals and visual observations of tens of crystals. A similar study has been carried out on phase IV [14].

Thermodynamically the equilibrium form of a crystal is determined by the Gibbs free energy [15]

$G=N \mu+\sum_{h k l} A_{h k l} \sigma_{h k l} \quad(T=0 \mathrm{~K})$,

where $N$ is the total number of particles, $\mu$ is the chemical potential per particle, $A_{h k l}$ the surface area of a face $(h k l)$ and $\sigma_{h k l}$ the surface free energy. $\sigma_{h k l}$ is related to the broken bond or attachment energy. Eq. (1) holds for $T=0 \mathrm{~K}$. At 
finite temperature an entropy term has to be added.

Because the Gibbs free energy has to be minimal, the sum part of eq. (1) has to be minimal for a certain number of particles, leading to an anisotropic growth form. This form can easily be found by constructing a so-called Gibbs-Wulff plot [16]. In this plot the orientation dependent surface free energy $\sigma$ is plotted versus orientation, resulting in a set of equilibrium forms. Different forms found are comparable with flat $(\mathrm{F})$ faces with steps or spirals. As the step free energy $\gamma>0$ (at $T=0 \mathrm{~K}$ ) sharp cusps in the Wulff plot will appear, corresponding to a set of flat faces. At $T>0 \mathrm{~K} \gamma$ will in general still be larger than 0 , resulting in the possibility for a certain face to appear. The cusps in the Wulff plot will not be as sharp, however.

We note that the crystallographic ideas used in this paper were already developed by Hartman and Perdok more than thirty years ago [17-19]. In this theory the concepts PBC (periodic bond chain) and $\mathrm{F}$ face play an essential role. $\mathrm{A} P B C$ is an uninterrupted path consisting of stoichiometric building units (direct space) connected through bonds, with the overall periodicity of a vector $[u v w]$ of the lattice, and F face is a face $(h k l)$ parallel to a connected net containing at least two mutually intersecting PBCs. So in this theory PBCs and connected nets are determined from the crystal structure.

The assumptions made in the present PBC analysis are that the first nearest neighbour bonds play an essential role in forming and ordening of a crystal structure and morphology. The interaction between the growth units and the mother phase and surface processes, e.g. surface reconstruction, as well as growth kinetics are as in the classical PBC analysis not taken into account. Thus the morphological analysis is factually carried out in a vacuum situation.

Furthermore a statistical mechanical approach will be applied which is first of all based on the concept of roughening transitions, resulting from Ising models, in which the whole interface crystal-solution is partitioned in equally shaped blocks (a type of Kossel crystal) which are either solid (s) or fluid (f). Three types of interaction occur between these blocks, a solid-solid, a fluid-fluid and a solid-fluid one, denoted as $\phi_{\mathrm{ss}}$, $\phi_{\mathrm{ff}}$ and $\phi_{\mathrm{sf}}$, with $\left\{\phi_{\mathrm{sf}}-\frac{1}{2}\left(\phi_{\mathrm{ss}}+\phi_{\mathrm{ff}}\right)\right\} / k T$ being the energy necessary to break or form a bond. These interactions account for the behaviour of a growth unit in the mother-phase and in the solid, as well as the interaction of the fluid. Furthermore, they are temperature dependent. The roughening transition implies that below a certain temperature $T_{\mathrm{r}}$, corresponding to the roughening transition, the edge free energy $\gamma$, of a step on a face $(h k l)$ is larger then zero and that above $T_{\mathrm{r}}, \gamma$ equals zero [20-23]. Below $T_{\mathrm{r}}$ surfaces are in essence flat and the crystal face grows by a layer mechanism, i.e. a two-dimensional nucleation mechanism or a spiral mechanism, because step or kink positions are energetically favoured. Above $T_{\mathrm{r}}$ the surface is rough and during growth the crystallographic orientation of the crystal face is more or less lost. When $\gamma=0$, growth units are free to incorporate at any position on the crystal surface [24]. In practice the so-called Ising temperature $T_{\mathrm{c}}$ is estimated, which is typically about $10 \%$ less than the real roughening temperature $T_{\mathrm{r}}[25$ 27].

In order to determine from the crystal structure which crystal faces will grow as flat faces, first the growth units have to be determined. In case of ammonium nitrate $\left(\mathrm{NH}_{4} \mathrm{NO}_{3}\right)$ we took the $\mathrm{NH}_{4}^{+}$ $(+)$ and $\mathrm{NO}_{4}^{-}(\Delta)$ ions. Next the bonds between the growth units have to be determined. As usual we limit ourselves to first nearest neighbour bonds. Thirdly we reduce the growth units to centres of gravity or points ( $\Delta$ and + ). These points together with the relations or bonds between the points are mostly equivalent to PBCs. They form a so-called crystal graph $[21,22]$. It can be seen as a topographic description of the crystal.

Now from the crystal graph the so-called connected nets have to be determined. These nets have an overall thickness $d_{h k l}$ defined as the interplanar distance and their occurrence is limited by the extinction conditions of the space group. Within such a net all growth units are connected to each other. For each net the total slice energy $E_{h k l}^{\text {slice }}$ is determined, being the sum of the energies of all bonds in a slice per stoichiometric unit and from this the attachment or the so-called broken 
bond energy $E_{h k l}^{\text {att }}$, which latter is a sum of the total of bonds per stoichiometric unit which lack due to a surface, because the total crystallization energy per stoichiometric unit $E_{\text {cryst }}$ is built up of $E_{h k l}^{\text {slice }}$ and $E_{h k l}^{\text {att }} . E_{h k l}^{\text {att }}$ divided by surface area of the stoichiometric unit for a face $(h k l)$, or equivalently multiplied by $d_{h k l} / V_{\text {stoich, }}$, where $V_{\text {stoich }}$ is the volume of one stoichiometric unit, will give a measure for the morphological importance (see below). This approximation for he equilibrium growth form holds at $T=0 \mathrm{~K}$. Connected nets show a roughening transition. When the temperature is below the roughening transition temperature the corresponding faces grow by a layer mechanism. The slowest growing faces will potentially occur on the growth forms. The Ising and roughening temperature of these faces are a measure for their stability.

In this paper we will consider two criteria to determine the relative morphological importance (MI) of faces namely if

$$
\begin{aligned}
& \left|E_{1}^{\text {ats }} d_{h k l}^{1}\right|<\left|E_{2}^{\text {att }} d_{h k l}^{2}\right| \text { or } \theta_{1}^{\mathrm{c}}>\theta_{2}^{\mathrm{c}} \text {, } \\
& \text { then } \mathrm{MI}_{1}>\mathrm{MI}_{2} .
\end{aligned}
$$

Here $\theta_{1}^{\mathrm{c}}$ is the dimensionless Ising (or roughening) temperature of face 1 .

In section 2 of this paper, the structure of $\mathrm{NH}_{4} \mathrm{NO}_{3}$ (III) is discussed and the first nearest neighbour bonds between the growth units $\mathrm{NH}_{4}^{+}$ and $\mathrm{NO}_{3}^{-}$are identified. In section 3, important crystallographic or $\mathrm{PBC}$ directions are presented. In section 4 , the connected nets, $E_{h k l}^{\text {att }}$ and $\theta^{\mathrm{c}}$ are determined. In section 5 , the theoretical growth forms are constructed using $E_{h k l}^{\text {att }} d_{h k l}$ (and $\theta^{\mathrm{c}}$ ), $E_{h k l}^{\text {att }}$ and $d_{h k l}$ as criteria for relative MI. In section 6 , the experimental morphology characterized by the crystal forms $\{h k l\}$ is presented. Finally, in section 7 , the results of theory and experiments are confronted with each other.

\section{Crystal structure and bonds}

The structure of $\mathrm{NH}_{4} \mathrm{NO}_{3}$ phase III, which is the thermodynamically stable structure for the

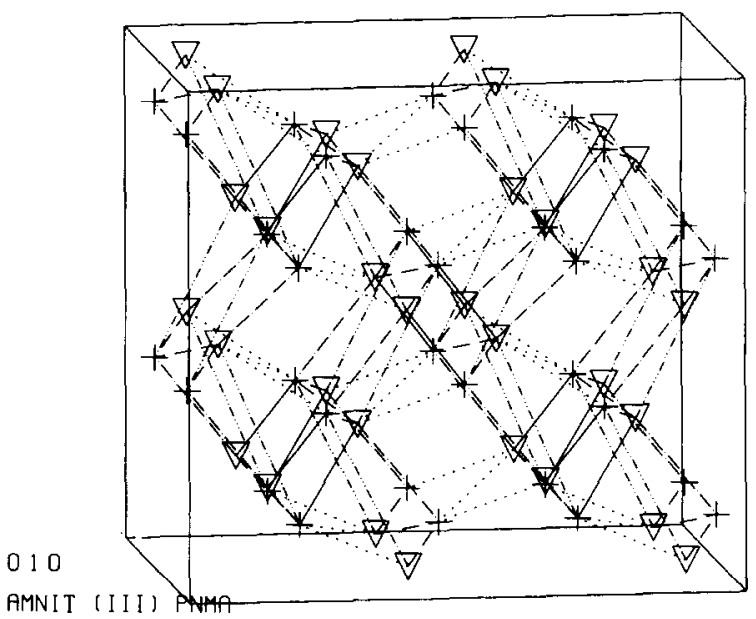

Fig. 1. [010] projection of eight elementary cells of $\mathrm{NH}_{4} \mathrm{NO}_{3}$ (III) with first nearest neighbour interactions: $(+)$ ammonium and $(\nabla)$ nitrate ions

temperature range $\approx 305<T<\approx 357 \mathrm{~K}$, has the orthorhombic space group Pnma,

$\mathrm{P} \frac{2_{1}}{\mathrm{n}} \frac{2_{1}}{\mathrm{n}} \frac{2_{1}}{\mathrm{n}} ; \mathrm{D}_{2 \mathrm{~h}}^{16}$ International Tables No. 62, $z=4$,

with crystal axes of length $(T=223 \mathrm{~K})[4,28-30]$ $a=7.716 \AA, b=5.845 \AA$ and $c=7.197 \AA$.

Extinction conditions are:

$0 k l: \quad k+l=2 n ; \quad k h 0: \quad h=2 n$.

We took the nitrogen as the centres of gravity of the growth units as being situated at the following positions [3,31]: $\mathrm{NH}_{4}^{+}(0.02 ; 0.25 ; 0.20), \mathrm{NO}_{3}^{-}$ $(0.31 ; 0.25 ; 0.59)$

In fig. 1 a projection of $\mathrm{NH}_{4} \mathrm{NO}_{3}$ (III) in the [010] direction is given. Eight elementary cells are shown with the first nearest neighbour bonds be-

Table 1

First nearest neighbour bonds in $\mathrm{NH}_{4} \mathrm{NO}_{3}$ (III)

\begin{tabular}{lllll}
\hline Index & $\begin{array}{l}\text { Length } \\
(\AA)\end{array}$ & $\begin{array}{l}\text { Line symbol } \\
\text { in fig. 1 }\end{array}$ & $\begin{array}{l}\text { Bond energy } \varphi_{i} \\
(\mathrm{~kJ} / \mathrm{mol})\end{array}$ & $\varphi_{i} / \varphi_{1}$ \\
\hline $\mathrm{a}$ & 2.64 & - & $\varphi_{1}=-540$ & 1.0 \\
$\mathrm{~b}$ & 3.30 & ---- & $\varphi_{2}=-432$ & 0.80 \\
$\mathrm{c}$ & 3.59 & $-\cdot-\cdot$ & $\varphi_{3}=-397$ & 0.74 \\
$\mathrm{~d}$ & 4.16 & $\cdots \cdots$ & $\varphi_{4}=-343$ & 0.64 \\
$\mathrm{e}$ & 4.93 & $-\cdots-$ & $\varphi_{5}=-289$ & 0.54 \\
\hline
\end{tabular}


tween the ions with opposite sign. Thus seven bonds per stoichiometric growth unit are present. Five different types of these bonds can be distinguished (table 1). Also the bond energies per mole are given based on the coulomb interaction between a positive ammonium and a negative nitrate ion.

\section{Projections}

As investigation off all connected nets leads to a set of PBCs within these nets. A rich variety of PBCs can be found. The results are summarized in table 2, where the PBCs are presented in their repetative units.

As can be seen, in certain directions more PBCs exist. Of these, the one with the lowest energy will probably, not necessarily, construct a slice in combination with PBCs of other directions. Using the crystallographic conventions, PBC directions are represented as great circles in the stereographic projection. The potential $\mathrm{F}$ faces parallel to connected nets are easily found as the intersections of at least two great circles. From the stereographic projection in fig. 2,25 possible $F$ forms remain, which are all investigated.

\section{Connected nets}

The face for which an unambiguous connected net can be constructed is then a stable $F$ face within the Hartman-Perdok approach. In fig. 3 these $\mathrm{F}$ forms are presented. There are thirteen forms resulting from the stereographic projection which are not connected, namely $\{024\},\{113\}$, $\{121\},\{122\},\{123\},\{132\},\{213\},\{214\},\{234\}$, $\{311\},\{312\},\{321\}$ and $\{412\}$.

In table 3 a summary of the different stable $F$ forms with bonds and slice energy $E_{h k l}^{\text {slice }}$ is given. For all the forms in table 3, the Ising temperature was calculated using the so-called Ising graph [23]. Not always it is possible to construct an Ising graph, e.g. due to "crossing bonds". In these cases an approximation has to be made. Usually a stronger and a weaker graph are made to obtain
Table 2

PBCs, bonds and energies, which form the connected nets

\begin{tabular}{|c|c|c|}
\hline $\begin{array}{l}\mathrm{PBC} \\
{[u v w]}\end{array}$ & Bonds per molecule & $\begin{array}{l}E \\
\left(10^{3} \mathrm{~J} / \mathrm{mol}\right)\end{array}$ \\
\hline$[001]_{1}$ & $\varphi_{3}+\varphi_{5}$ & -688 \\
\hline$[001]_{2}$ & $\frac{1}{2}\left(\varphi_{1}+\varphi_{2}+\varphi_{3}+\varphi_{4}\right)$ & -857 \\
\hline$[001]_{3}$ & $\frac{1}{2}\left(\varphi_{1}+\varphi_{2}+\varphi_{3}+\varphi_{4}\right)$ & -857 \\
\hline$[100]_{1}$ & $\varphi_{1}+\varphi_{3}$ & -939 \\
\hline$[100]_{2}$ & $\varphi_{1}+\varphi_{5}$ & -831 \\
\hline$[100]_{3}$ & $\varphi_{2}+\varphi_{4}$ & -776 \\
\hline$[100]_{4}$ & $\frac{1}{2}\left(\varphi_{1}+\varphi_{2}+\varphi_{3}+\varphi_{4}\right)$ & -857 \\
\hline$[100]_{5}$ & $\varphi_{2}+\varphi_{4}$ & -776 \\
\hline$[100]_{6}$ & $\varphi_{2}+\varphi_{4}$ & -776 \\
\hline$[100]_{7}$ & $\frac{1}{2}\left(\varphi_{1}+\varphi_{2}+\varphi_{3}+\varphi_{4}\right)$ & -857 \\
\hline$[010]_{1}$ & $2 \varphi_{2}$ & -866 \\
\hline$[010]_{2}$ & $2 \varphi_{4}$ & -687 \\
\hline$[\overline{1} 01]_{1}$ & $\varphi_{1}+1 / 2\left\{\varphi_{3}+\varphi_{5}\right\}$ & -890 \\
\hline$[\overline{1} 01]_{2}$ & $\frac{1}{2}\left\{\varphi_{1}+\varphi_{2}+\varphi_{3}+\varphi_{4}\right\}$ & -857 \\
\hline$[\overline{1} 01]_{3}$ & $\frac{1}{2}\left\{\varphi_{1}+\varphi_{2}+\varphi_{4}+\varphi_{5}\right\}$ & -803 \\
\hline$[01 \overline{1}]_{1}$ & $\varphi_{2}+\varphi_{3}$ & -831 \\
\hline$[01 \overline{1}]_{2}$ & $\varphi_{1}+\varphi_{4}$ & -884 \\
\hline$[01 \overline{1}]_{3}$ & $\frac{1}{2}\left\{\varphi_{1}+\varphi_{2}+\varphi_{3}+\varphi_{4}\right\}$ & -857 \\
\hline$[01 \overline{1}]_{4}$ & $\frac{1}{2}\left\{\varphi_{1}+\varphi_{2}+\varphi_{3}+\varphi_{4}\right\}$ & -857 \\
\hline$[01 \overline{1}]_{5}$ & $\varphi_{2}+\varphi_{5}$ & -723 \\
\hline$[\overline{1} 10]_{l}$ & $\frac{1}{2}\left\{\varphi_{1}+\varphi_{2}+\varphi_{3}+\varphi_{4}\right\}$ & -857 \\
\hline$[\overline{1} 10]_{2}$ & $\varphi_{2}+\varphi_{4}$ & -776 \\
\hline$[\overline{1} 10]_{3}$ & $\frac{1}{2}\left\{\varphi_{1}+\varphi_{2}+\varphi_{3}+\varphi_{4}\right\}$ & -857 \\
\hline$[\overline{1} 10]_{4}$ & $\frac{1}{2}\left\{\varphi_{1}+\varphi_{2}+\varphi_{3}+\varphi_{4}\right\}$ & -857 \\
\hline$[\overline{1} 10]_{5}$ & $\frac{1}{2}\left\{\varphi_{1}+\varphi_{2}+\varphi_{3}+\varphi_{4}\right]$ & -825 \\
\hline$[\overline{1} 1 \overline{1}]_{1}$ & $\frac{1}{2}\left\{\varphi_{1}+\varphi_{2}+\varphi_{3}+\varphi_{4}\right]$ & -857 \\
\hline$[\overline{1} 1 \overline{1}]_{2}$ & $\frac{1}{3}\left\{\varphi_{1}+\varphi_{2}+\varphi_{3}+\varphi_{4}\right\}$ & -860 \\
\hline$[11 \overline{1}]_{1}$ & $\frac{1}{2}\left\{\varphi_{1}+\varphi_{2}+\varphi_{3}+\varphi_{4}\right\}$ & -857 \\
\hline$[11 \overline{1}]_{2}$ & $\frac{1}{2}\left\{\varphi_{1}+\varphi_{2}+\varphi_{4}+\varphi_{5}\right\}$ & -803 \\
\hline$[\overline{1} 11]_{1}$ & $\frac{1}{2}\left\{\boldsymbol{\varphi}_{1}+\boldsymbol{\varphi}_{2}+\boldsymbol{\varphi}_{\mathbf{4}}+\boldsymbol{\varphi}_{5}\right\}$ & -803 \\
\hline$[\overline{1} 11]_{2}$ & $\frac{1}{2}\left\{\varphi_{1}+\varphi_{2}+\varphi_{4}+\varphi_{5}\right\}$ & -803 \\
\hline$[\overline{1} 11]_{3}$ & $\frac{1}{2}\left\{\varphi_{1}+\varphi_{2}+\varphi_{3}+\varphi_{4}\right\}$ & -857 \\
\hline$[\overline{1} 11]_{4}$ & $\frac{1}{3}\left\{2 \varphi_{1}+2 \varphi_{2}+\varphi_{3}+\varphi_{5}\right\}$ & -878 \\
\hline$[\overline{1} 11]_{5}$ & $\frac{1}{3}\left\{2 \varphi_{1}+\varphi_{2}+\varphi_{4}+2 \varphi_{5}\right\}$ & -779 \\
\hline$[\overline{1} 20]_{1}$ & $\varphi_{2}+\varphi_{4}$ & -776 \\
\hline$[02 \overline{1}]_{1}$ & $\frac{1}{3}\left\{2 \varphi_{1}+2 \varphi_{2}+2 \varphi_{4}\right\}$ & -878 \\
\hline$[02 \overline{1}]_{2}$ & $\frac{1}{3}\left\{2 \varphi_{1}+2 \varphi_{2}+2 \varphi_{4}\right]$ & -878 \\
\hline$[20 \overline{1}]_{1}$ & $\frac{1}{4}\left\{\varphi_{1}+3 \varphi_{2}+\varphi_{3}+3 \varphi_{4}\right\}$ & -808 \\
\hline$[\overline{1} 2 \overrightarrow{1}]_{1}$ & $\frac{1}{3}\left\{2 \varphi_{1}+2 \varphi_{2}+2 \varphi_{4}\right\}$ & -878 \\
\hline$[\overline{1} 2 \overline{1}]_{2}$ & $\frac{1}{3}\left\{2 \varphi_{1}+2 \varphi_{2}+2 \varphi_{4}\right\}$ & -878 \\
\hline$[\overline{1} 2 \overline{1}]_{3}$ & $\frac{1}{3}\left\{2 \varphi_{1}+2 \varphi_{2}+2 \varphi_{4}\right\}$ & -878 \\
\hline
\end{tabular}

an estimate of the real Ising temperature. We made several approximations for the strongest net $\{101\}$. 


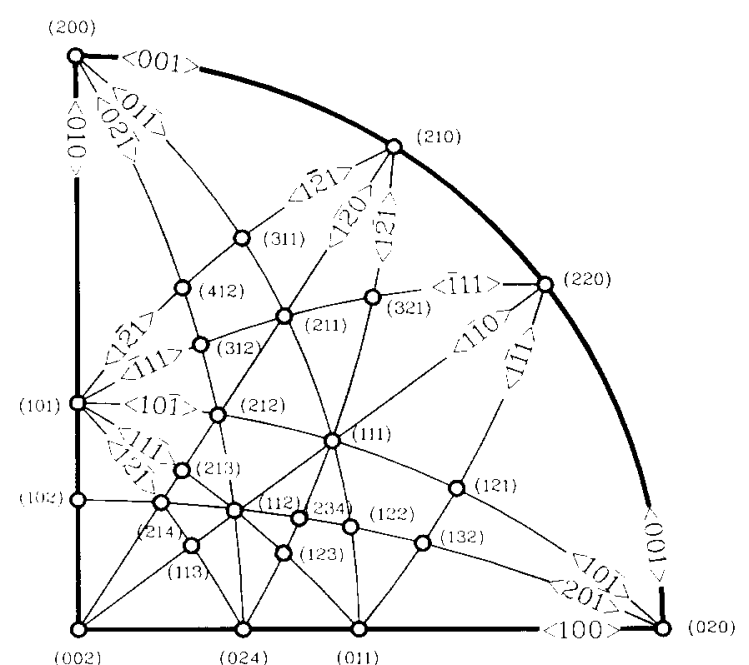

Fig. 2. Stereographic projection of $\mathrm{PBCs}$ in $\mathrm{NH}_{4} \mathrm{NO}_{3}$ (III) crystals. Potential $F$ faces are intersections of two or more PBCs.

From another study, the roughening transition temperature of the $\{111\}$ faces could be estimated as $440 \pm 20 \mathrm{~K}$ [32]. This result is used to estimate the roughening temperatures of the other faces.

If more than one alternative connected net $(\mathrm{F}$ form $\{h k l\})$ could be constructed, we only took the one with the strongest connected net and/or highest Ising temperature. In table 4 these Ising temperatures are presented. Note the different
Table 4

Ising temperatures of the connected nets as well as the relative temperatures; note the difference with table 3

\begin{tabular}{llll}
\hline Form & No. & $\theta_{c}(h k l) / \theta_{c, \max }$ & $\begin{array}{l}T_{\text {c,est }} \\
(\mathrm{K})\end{array}$ \\
\hline$\{101\}$ & 1 & $0.89-1.0$ & $600-680$ \\
$\{011\}$ & 2 & 0.77 & 520 \\
$\{200\}$ & 3 & 0.70 & 470 \\
$\{210\}$ & 4 & 0.66 & 450 \\
$\{111\}$ & 5 & $0.62-0.66$ & $440\left(T_{\mathrm{r}}\right)$ \\
$\{102\}$ & 6 & 0.60 & 410 \\
$\{211\}$ & 7 & 0.58 & 390 \\
$\{002\}$ & 8 & 0.50 & 340 \\
$\{020\}$ & 9 & 0.48 & 320 \\
$\{212\}$ & 10 & 0.39 & 260 \\
$\{220\}$ & 11 & 0.37 & 250 \\
$\{112\}$ & 12 & 0.36 & 240 \\
\hline
\end{tabular}

ordering as compared to table 3. Because $\mathrm{NH}_{4}$ $\mathrm{NO}_{3}$ (III) is only stable at temperatures above 305 $\mathrm{K}$, the $\{212\},\{220\}$ and $\{112\}$ faces are not expected on the growth forms, $\{020\}$ being doubtful.

\section{Morphological importance and theoretical growth forms}

The morphological importance (MI) of a crystal face is a measure for the chance of appearance

Table 3

Crystal forms of $\mathrm{F}$ type of $\mathrm{NH}_{4} \mathrm{NO}_{3}(\mathrm{III}), E_{h k l}^{\text {slice }}, d_{h k l}$ and $\xi$ are given; $\xi=E_{h k l}^{\text {slice }} / E^{\text {cryst }}$; absolute values of energies are given: $E^{\text {cryst }}=E_{h k l}^{\text {slice }}+E_{h k l}^{\text {att }}=(\varphi+2 \varphi+\varphi+2 \varphi+\varphi)=-2.78 \mathrm{MJ} / \mathrm{mol}$

\begin{tabular}{lllllll}
\hline $\begin{array}{l}\text { Crystal } \\
\text { form } \\
\{h k l\}\end{array}$ & $\begin{array}{l}\text { Bonds in slice } \\
\text { per molecule }\end{array}$ & $\begin{array}{l}E_{h k l}^{\text {slice }} \\
(\mathrm{MJ} / \mathrm{mol})\end{array}$ & $\xi$ & $\begin{array}{l}d_{h k l} \\
(\AA)\end{array}$ & $\begin{array}{l}E_{h k l}^{\text {att }} \\
(\mathrm{MJ} / \mathrm{mol})\end{array}$ & $\left.\begin{array}{l}d_{h k l} E_{h k l}^{\text {att }} \\
(\mathrm{MJ} \AA \\
\AA\end{array} / \mathrm{mol}\right)$
\end{tabular}


and for the size of that face. The theoretical morphology can be determined using different criteria. In a first approach the interplanar distance is a fairly good measure for the morphological importance, because the energy content in a layer $d_{h k l}$ is one determining factor [33]. But also, the faces

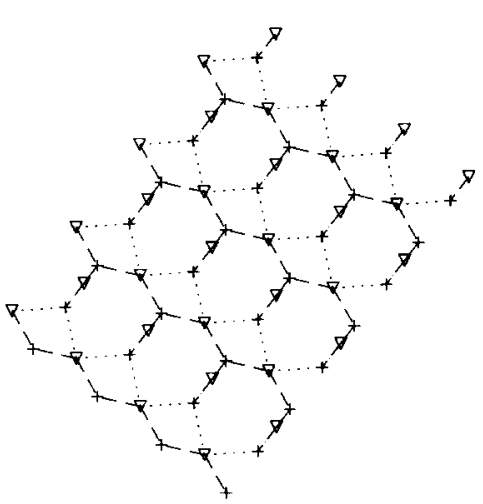

AMNIT IIII) PNMA 002

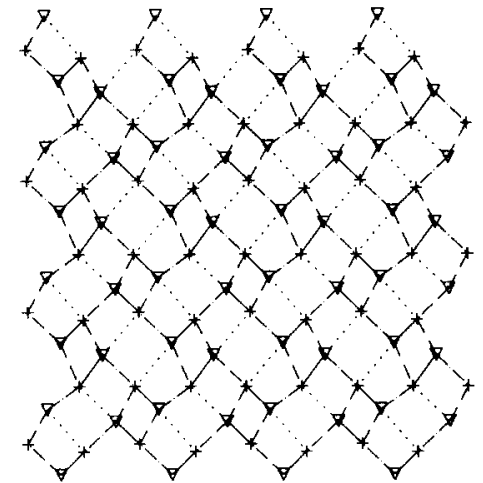

AMNIT (III) PNMA OLI

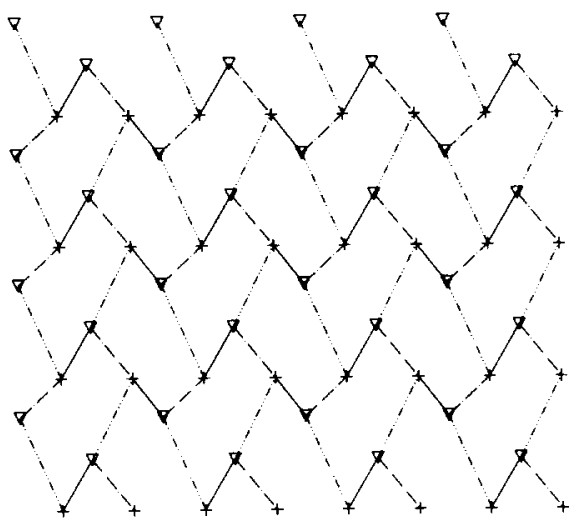

AMNIT I III] PNMA 020

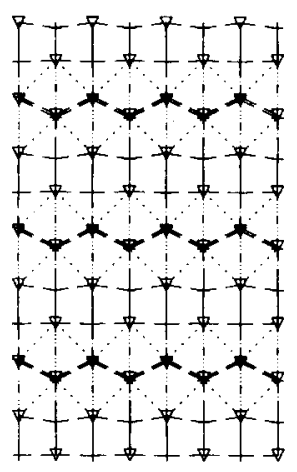

PMNIT : III] PNMA 10 :

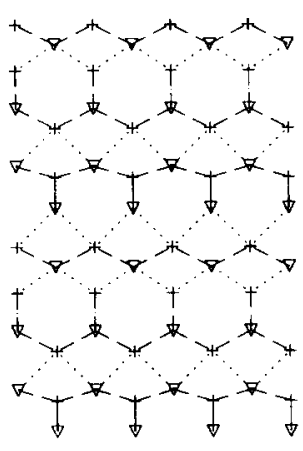

AMNIT (III) PNMA 102

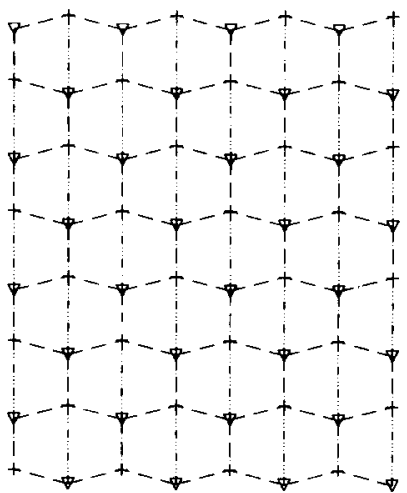

AMNIT (III) PNMA 200

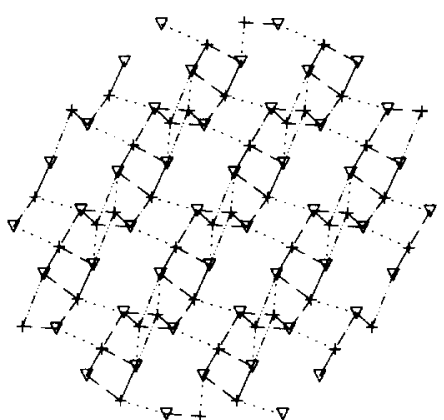

AMNIT IIII) PNMA 1111

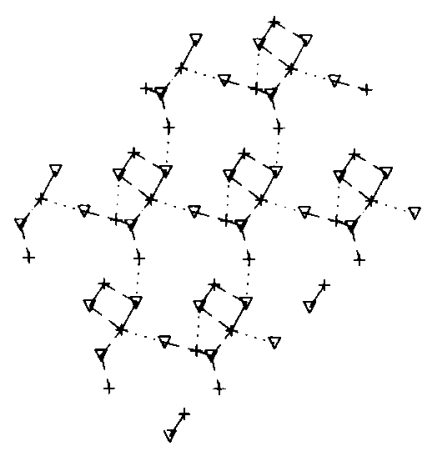

AMNIT IIII] PNMA 112

Fig. 3. F faces of $\mathrm{NH}_{4} \mathrm{NO}_{3}$ (III). All connected nets are shown perpendicular to $(h k l)$. 


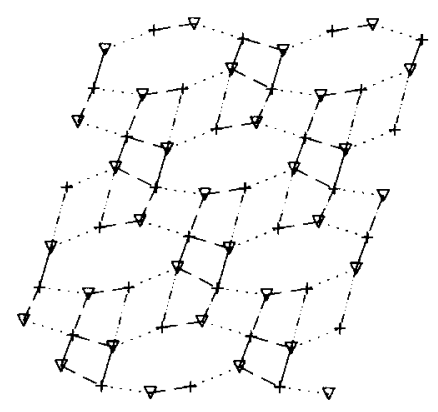

FMNIT (III) PNMA 211

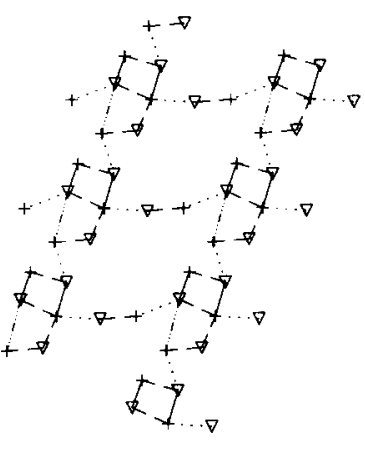

AMNIT (III) PNMA 212

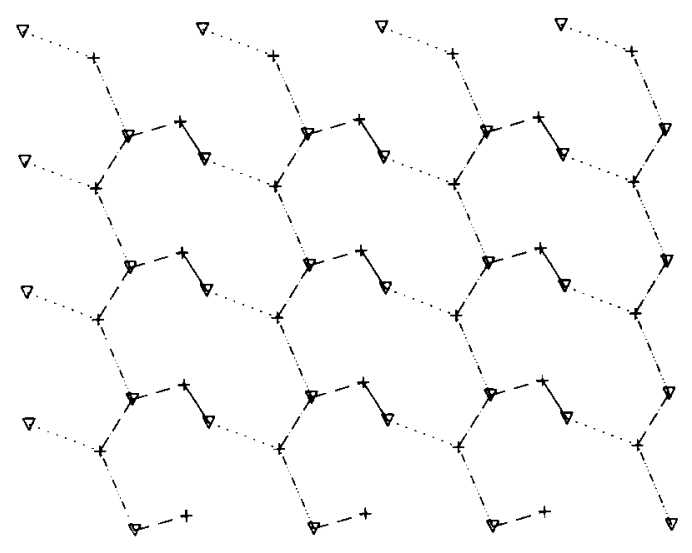

AMNIT I I I I ) PNMA 220

Fig. 3 (continued).

have to be connected, thus $E_{h k l}^{\text {slice }}$ of a connected net is a better approach. In a Gibbs-Wulff construction the surface free energy $E_{h k l}^{\text {att }} d_{h k l} / V_{\text {stoich }}$ is the measure for the MI. $E_{h k l}^{\text {slice }}$ and $E_{h k l}^{\text {att }}$ are determined by the Hartman-Perdok theory. Furthermore, also the way a net is connected should be taken into account, which is done by determining $\theta_{\mathrm{c}}$. Experimentalists often use $E_{h k l}^{\text {att }}$ to construct growth forms of three-dimensional Wulff plots [34]. Although this ad hoc relation cannot be justified from theory, in practice it gives quite

Table 5

Morphological importance based on the interplanar distance $d_{h k l}$, the roughening temperature $\theta_{\mathrm{c}}$ and the attachment energy $E_{h k l}^{\text {att }}$ or $E_{h k l}^{\text {att }} d_{h k l}, \mathrm{MI}_{\text {theor }}$ being determined from $E_{h k l}^{\text {att }} d_{h k l}$ for a flat face ( $f$ : flat faces; $r$ : rough faces); the numbers indicate MI ranks based on tables 3 and 4

\begin{tabular}{|c|c|c|c|c|c|}
\hline Form & $\begin{array}{l}d_{h k l} \\
(\AA)\end{array}$ & $\begin{array}{l}\theta_{\mathrm{c}}^{\text {est }} \\
(\mathbf{K}) \\
\end{array}$ & $\begin{array}{l}E_{h k l}^{\mathrm{att}} \\
(\mathrm{MJ} / \mathrm{mol})\end{array}$ & $\begin{array}{l}E_{h k l}^{\mathrm{att}} d_{h k l} \\
(\mathrm{MJ} \AA / \mathrm{mol})\end{array}$ & $\mathrm{MI}_{\text {theor }}$ \\
\hline$\{101\}$ & 1 & f & 1 & 1 & 1 \\
\hline$\{211\}$ & 8 & f & 7 & 4 & 4 \\
\hline$\{210\}$ & 7 & f & 5 & 7 & 7 \\
\hline$\{111\}$ & 3 & $\mathrm{f}$ & 3 & 8 & 8 \\
\hline$\{102\}$ & 6 & $\mathrm{f}$ & 6 & 9 & 9 \\
\hline$\{011\}$ & 2 & $\mathrm{f}$ & 2 & 10 & 10 \\
\hline$\{200\}$ & 4 & $\mathrm{f}$ & 4 & 11 & 11 \\
\hline$\{002\}$ & 5 & $\mathrm{f}$ & 8 & 12 & 12 \\
\hline$\{020\}$ & 9 & $\mathrm{f}$ & 9 & 6 & 6 \\
\hline$\{212\}$ & 11 & $r$ & 11 & 2 & $r$ \\
\hline$\{220\}$ & 12 & $r$ & 12 & 3 & $r$ \\
\hline$\{112\}$ & 10 & r & 10 & 5 & $r$ \\
\hline
\end{tabular}

satisfactory results [35-39]. The reason why the growth form obeys the $E_{h k l}^{\text {att }}$ dependence rather than that of $E_{h k l}^{\text {att }} d_{h k l}$ may be found in the following relation which describes the growth velocity $R_{h k l}$ perpendicular to a face $(h k l)$ [34]:

$R_{h k l}^{\ddagger} / R_{h k l} \sim E_{h k l}^{\mathrm{att} \ddagger} / E_{h k l}^{\mathrm{att}}$.

In table 5 all these different approaches are gathered. The agreement of these four methods is rather poor.

In fig. 4 we present the growth forms, taking the relative rates of growth proportional to $E_{h k l}^{\text {att }} d_{h k l}, E_{h k l}^{\text {att }}$ and $d_{h k l}$.

\section{Experimental growth forms}

To compare the results of the $\mathrm{PBC}$ analysis of $\mathrm{NH}_{4} \mathrm{NO}_{3}$ (III) with crystal habits, crystals had to be grown. Although much crystallographic work has been done on the material and from this work rough ideas about the growth forms could be obtained, up till now hardly any data concerning the morphology are available, while for $\mathrm{NH}_{4} \mathrm{NO}_{3}$ (IV) already in 1906 the growth form was known [40]. This is possibly due to the fact that it is difficult to grow these crystals from solution and to manipulate them. In practice, the chances that nuclei get formed are high and thus it is difficult to grow large crystals, $\sigma$ has to be small $(\sim 0.2 \%)$ 

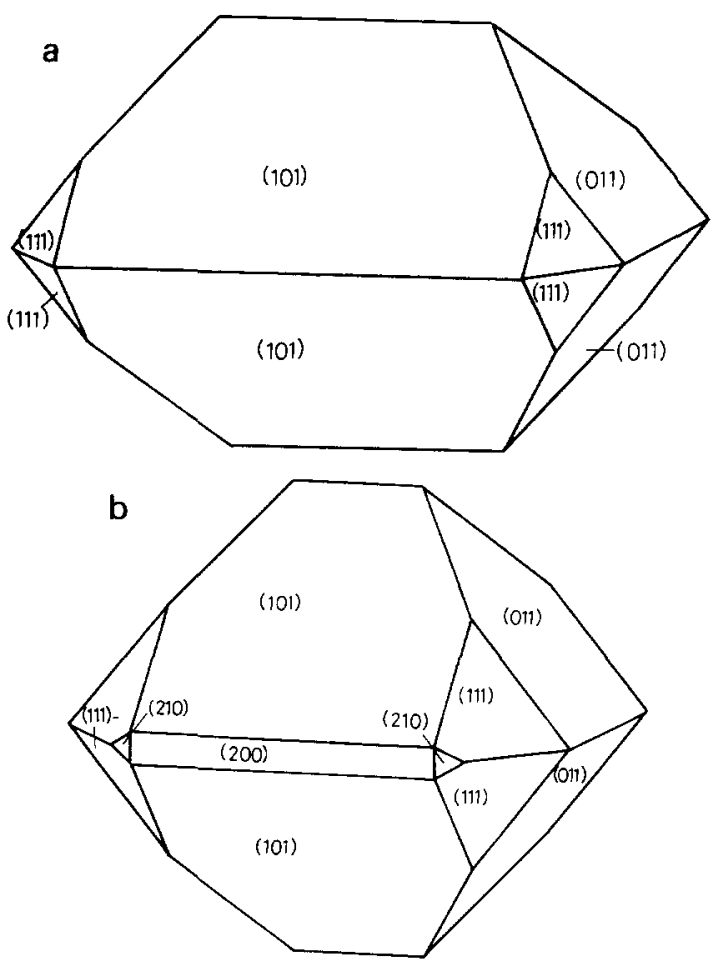

。

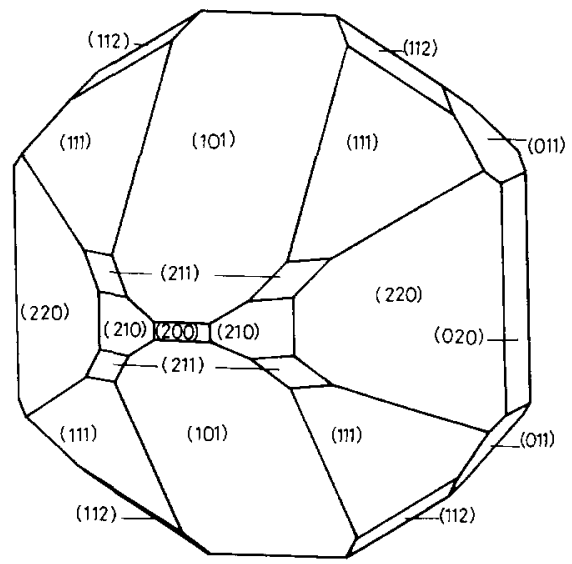

Fig. 4. Growth forms of $\mathrm{NH}_{4} \mathrm{NO}_{3}$ (III) crystal based on $E_{h k l}^{\text {att }}$, $d_{h k l}$ and $E_{h k l}^{\text {att }} d_{h k l}$.

to prevent needle like growth forms; in order to obtain better results the solution was stirred. Also the form of the crystal gave rise to problems, because they easily bend. Crystals of phase III are hygroscopic, not stable under room temperature conditions and turn white within a short period. This makes it hard to preserve them. Last of all it
Table 6

Experimental crystals forms and MIs; for normalization, see text

\begin{tabular}{lllc}
\hline Form & \#Normalized & $\mathrm{MI}_{\exp }$ & $\mathrm{MI}_{\text {theor }}$ \\
\hline$\{101\}$ & 14 & 1 & 1 \\
$\{111\}$ & 10.25 & 2 & 8 \\
$\{200\}$ & 9 & 3 & 11 \\
$\{011\}$ & 3.5 & $4 / 5$ & 10 \\
$\{002\}$ & 3 & $4 / 5$ & 12 \\
$\{220\}$ & 2 & 6 & $\mathrm{r} / 2$ \\
$\{210\}$ & - & $7 / 12$ & 7 \\
$\{102\}$ & - & $7 / 12$ & 9 \\
$\{211\}$ & - & $7 / 12$ & 4 \\
$\{020\}$ & & $7 / 12$ & 6 \\
$\{212\}$ & - & $7 / 12$ & $\mathrm{r} / 3$ \\
$\{112\}$ & - & $7 / 12$ & $\mathrm{r} / 5$ \\
\hline
\end{tabular}

was necessary to do the goniometer experiments in a temperature controlled environment above 305 K.

Some ten crystals were grown at about $315 \mathrm{~K}$ in a stirred solution with typical sizes of a few $\mathrm{mm}^{3}$, large enough to carry out optical goniometer experiments. Table 6 gives the results of these experiments.

A typical growth form of $\mathrm{NH}_{4} \mathrm{NO}_{3}$ (III) is given in fig. 5.

The experimental MI is determined only by number of appearances. Observing the crystal

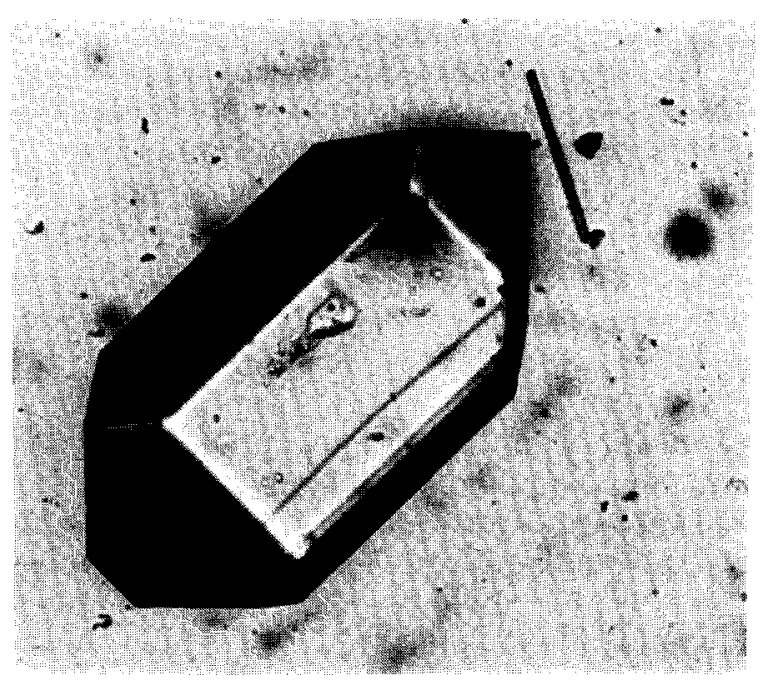

Fig. 5. Experimental growth forms of $\mathrm{NH}_{4} \mathrm{NO}_{3}$ (III) crystals. 
form, it is clear that the $\{101\}$ faces are by far the largest. $\{111\}$ faces are quite small, but they always appear, in contrast to $\{200\},\{011\}$ and $\{002\}$ faces which are larger, but are not always present. The $\mathrm{MI}_{\text {exp }}$ for a form is normalized, in order to take account of the chance that a certain face appears being correlated to the number of equivalent growth faces in that form.

\section{Discussion}

In the preceding sections we derived the crystal habit. The correspondence between MI predicted by theory and experimentally found is poor. All important predicted $F$ faces were found, however, except for the $\{220\}$ form which is found but expected to be roughened. As noticed, the Ising
RMNIT (III) PNMA $0 \quad 0 \quad 1$

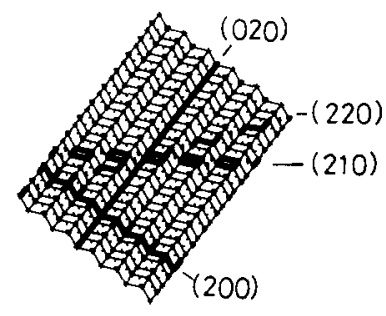

AMNIT (III) PNMA 1000

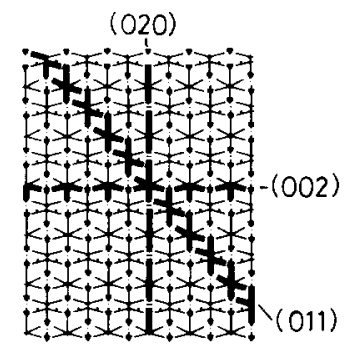

AMNIT (III) PNMA 0 1 0

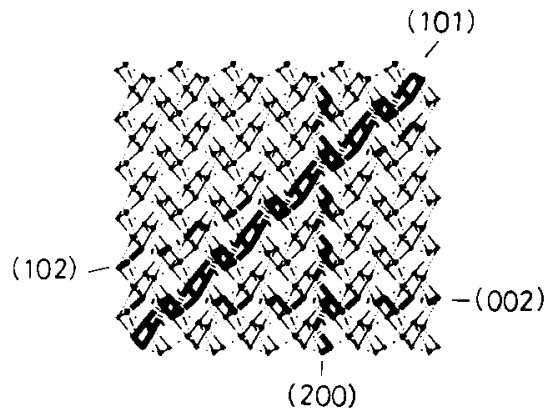

AMNIT IIIIJ PNMA 110

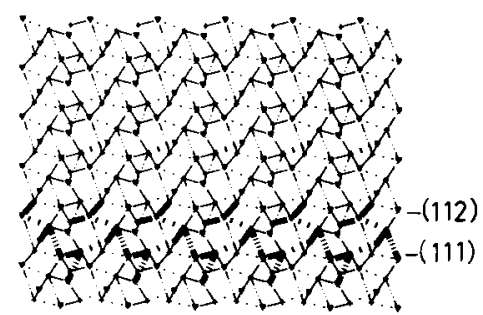

AMNII (III) PNMA 2110

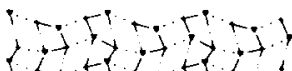

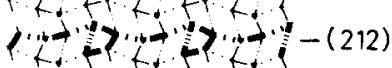

<.t.

aty $y+7+1$

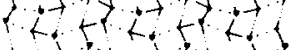

$6+7+5+4+3$

Fig. 6. Side views of the 12 connected nets, represented by thick rows. Also given are the perpendicular faces, in which the side views are projected. 
temperature is smaller than the roughening transition. Also the estimate of $T_{\mathrm{r} 111}$ is not very accurate. These two errors might account for the difference found.

On the other hand, the morphology of crystals is determined to a great extent by the growth properties, such as growth units, solvent and impurities. The following is a discussion of the different faces and their sensitivity to impurities. In recent qualitative experiments we have found that the growth form of $\mathrm{NH}_{4} \mathrm{NO}_{3}$ (III) is sensitive to positive ions. We observed that mainly the three valent ions effect the growth and not $\mathrm{NH}_{4}^{+}$or $\mathrm{H}^{+}$ (i.e. $\mathrm{pH}$ ). The morphology didnot change radically, but the constitional supercooling clearly became larger. This discussion is always rather tricky, because the exact influence of an impurity to for instance a face with a net possitive charge is unclear. The interaction between solvent and a face and/or impurities might lead to the opposite of what is expected at first. Nevertheless, the influence of impurities blocking spirals and steps can explain the asymmetry in sizes of faces belonging to one form. Observations of the expermentally grown crystals show that of the $\{011\}$, $\{002\}$ and $\{220\}$ forms normally only one face is present, probably due to impurities. Also crystal faces might become smaller, e.g. $\{111\}$ faces. In fact this is the case for many crystals, see e.g. fig. 5.

It is clear that the $\{101\}$ faces are the most important, in these faces most of the ions have six bonds per stoichiometric unit, with both positive and negative ions in the top layer, the negative ions a bit more to the surface, and therefore they are relatively sensitive to impurities (fig. 6). These faces will dominate the growth form, probably even more than predicted with the before mentioned criteria, due to the positive metal impurities. Then there is a group of moderately important faces. Of these the $\{011\}$ faces are the strongest, but because of a negatively charged top layer positively charged impurities like metal-ions can block the spirals and steps easily (fig. 6). Thus the $\{011\}$ form will also be more important than predicted by theory. This also holds for the $\{111\}$ faces, but in a lesser extent. The $\{200\},\{210\}$ and $\{211\}$ faces have mixed top-layers and should be fairly insensitive to impurities. The $\{102\}$ faces have a net positive charged top layer and a net negative charged layer. The positive charged layer might be sensitive to negative impurities, but an influence of this type of impurity is seldomly found.

The $\{002\}$ faces have one top-layer with negative ions and adsorbed positive impurities will slow down growth. The $\{020\}$ faces are very special. All ions are situated in the $\mathrm{m}$ mirror plane. Thus this will be a very flat layer, therefore no effect is expected.

The $\{212\}$ and $\{112\}$ layers are not very sensitive to impurities, whilst the $\{220\}$ faces are either sensitive to negative or to positive ions [41-43].

As the crystal habit is concerned the best agreement between experiment and theory is found when using $d_{h k l}$ or to a somewhat lesser extent $E_{h k l}^{\text {att }}$. The theoretical growth form can be somewhat improved taking into account the influence of impurities. This discrepancy between theory and experiment will be subject of further study.

\section{Acknowledgements}

The authors acknowledge the financial support of the Netherlands Organisation for Scientific Research (NWO/SON) as well as DSM, The Netherlands, for stimulating discussions. We wish to thank Dr. H. Meekes for fruitful remarks concerning the manuscript.

\section{References}

[1] J. Whetstone, J. Ind. Chem. 44 (1952) 2663.

[2] L. Phoenix, Brit. Chem. Eng. 11 (1966) 34.

[3] T.H. Goodwin and J. Whetstone, J. Chem. Soc. (1947) 1455.

[4] S.B. Hendricks, E. Posnjak and F.C. Kracek, J. Am. Chem. Soc. 54 (1932) 2766.

[5] A. Théorêt and C. Sandorfy, Can. J. Chem. 42 (1964) 57.

[6] R.N. Brown and A.C. McLaren, Proc. Royal Soc. (London) A266 (1962) 329.

[7] I. Konkoly-Thege, J. Therm. Anal. 12 (1977) 197.

[8] A. Sieverts and H. Theberath, Z. Anorg. Chem. 144 (1925) 61.

[9] D. Fatu, S. Fatu and E. Segal, Rev. Rom. Chim. 18 (1978) 1709. 
[10] O.S. Novikova, Y.V. Tsekhanskaya, O.I. Titova and T.I. Gontarenko, Russ. J. Phys. Chem. 51 (1977) 152.

[11] H. Maurus, Compt. Rend. (Paris) C276 (1973) 285.

[12] F. Wolf, K. Benecke and H. Fürtig, Z. Physik. Chem. 249 (1972) 274.

[13] F. Wolf and W. Scharne, Zesz. Nauk. Uniw. Pozaniu Mat. Fiz. Chem. 11 (1967) 3, 25, 44, 66.

[14] M.H.J. Hottenhuis, L.J.P. Vogels, P. Bennema and M.C. Elwenspoek, J. Crystal Growth, submitted.

[15] M. Volmer, Kinetik der Phasenbildung (Steinkopff, Leipzig, 1939).

[16] G. Wulff, Z. Krist. 34 (1901) 449.

[17] P. Hartman and W.G. Perdok, Acta Cryst. 8 (1955) 49 , $521,525$.

[18] P. Hartman, Z. Krist. 119 (1963) 65.

[19] P. Hartman, in: Crystal Growth: An introduction, Ed. P. Hartman (North Holland, Amsterdam, 1973) p. 367.

[20] K.A. Jackson, in: Crystal Growth, Ed. H.S. Peiser (Pergamon, Oxford, 1967) p. 17.

[21] P. Bennema and J.P. van der Eerden, in: Morphology of Crystals, Part A: Fundamentals, Ed. I. Sunagawa (Kluwer, Dordrecht, 1987).

[22] P. Bennema, in: Morphology and Growth Unit of Crystals, Proc. Oji Intern. Seminar on Morphology and Growth of Crystals, Zao Hot Springs, August 1985, Ed. I. Sunagawa (Terra, Tokyo, 1989) pp. 219-245.

[23] J.J.M. Rijpkema, H.J.F. Knops, P. Bennema and J.P. van der Eerden, J. Crystal Growth 61 (1982) 295.

[24] J.P. van der Eerden, C. van Leeuwen, P. Bennema, W.L. van de Kruk and B.P. Veltman, J. Appl. Phys. 48 (1977) 2124.

[25] J.D. Weeks and G.H. Gilmer, in: Advances in Chemical Physics, Eds. I. Prigogine and S.A. Rice (Wiley, New York, 1979) p. 157.

[26] H. Beijeren, Commun. Math. Phys. 40 (1975) 1.

[27] J.P. van der Eerden, P. Bennema and T.A. Cherepanova, Progr. Crystal Growth Characterization 1 (1978) 219.

[28] N.F.M. Henry and L. Lonsdale, International Tables for
X-Ray Crystallography, Vol. 1 (Kynoch, Birmingham. 1976).

[29] C.S. Choi, H.J. Prask and E. Prince, J. Appl. Cryst. 13 (1980) 403

[30] T. Seiyama and N. Yamazoe, J. Crystal Growth 2 (1968) 255.

[31] R.W.G. Wyckoff, Crystal Structures, Vol. 2 (Wiley-Interscience, New York, 1964) p. 368.

[32] L.J.P. Vogels, H.A.M. Marsman, M.A. Verheijen, P. Bennema and M. Elwenspoek, J. Crystal Growth 100 (1990) 439.

[33] J.D.H. Donnay and D. Harker, Am. Mineralogist 22 (1937) 446.

[34] P. Hartman and P. Bennema, J. Crystal Growth 49 (1980) 145.

[35] L. Addadi, Z. Berkovitch-Yellin, J. Weissbuch et al., Mol. Crystals Liquid Crystals 96 (1983) 1.

[36] L. Addadi, Z. Berkovitch-Yellin, N. Domb, E. Gati, M. Lahav and L. Leiserowitz, Nature 296 (1982) 21.

[37] Z. Berkovitch-Yellin, L. Addadi, M. Idelson, M. Lahav and L. Leiserowitx, Angew. Chem. (Suppl.) 94 (1980) 1336.

[38] Z. Berkovitch-Yellin, L. Addadi, M. Idelson, L. Leiserowitz and M. Lahav, Nature 296 (1982) 27.

[39] K.J. Roberts, L.A.M.J. Jetten and P. Bennema, Inst. of Chem. Eng., North Western Branch Papers No. 3 (1985) 2.1.

[40] P. Groth, Chemische Kristallographie, Part 2 (Leipziger Verlag, 1908) p. 76.

[41] M.H.J. Hottenhuis and C.B. Lucasius, J. Crystal Growth 78 (1986) 379.

[42] N. Cabrera and D.A. Vermilyea, in: Growth and Perfection of Crystals, Eds. R.H. Doremus, B.W. Roberts and D. Turnbull (Wiley, New York, 1958) p. 393.

[43] B. Dam, E. Polman and W.J.P. van Enckevort, in: Industrial Crystallization 84, Eds. S.J. Jančić and E.J. de Jong (Elsevier, Amsterdam, 1984) p. 97. 